\title{
SOME RELATIONS BETWEEN FUNCTIONALS ON BOUNDED REAL SQUENCES
}

\author{
SEYHMUS YARDIMCI
}

\begin{abstract}
In this paper, we mainly concern with the functionals $L^{* *}$ and $l^{* *}$ defined on bounded real sequences and give some inequalities between these functionals.
\end{abstract}

\section{INTRODUCTON}

If $T=\left(t_{n k}\right)$ is an infinite matrix with real entries, and if $x=\left(x_{k}\right)$ is a sequence of real numbers, then $T x$ denotes the transformed sequence whose $n$-th term is given by $(T x)_{n}=\sum_{k=1}^{\infty} t_{n k} x_{k}$. In order to investigate the effect of such transformations upon the derived set, Knopp [5] introduced the idea of the core $(\mathcal{K}$-core) of a sequence and proved the well-known Core Theorem. That theorem asserts that $\mathcal{K}$-core $\{T x\} \subseteq \mathcal{K}$-core $\{x\}$, whenever $T x$ exists for the nonnegative regular matrix $T$. Some variants of the Core Theorem may be found in [2], [9], [10], [12].

Considering the method of almost convergence Loone [6] and Das [2] introduced the Banach core $(\mathcal{B}$-core) of a bounded sequence and proved some analogues of the assertions for the $\mathcal{K}$-core (see also [4], [10], [12], [13]).

Before proceeding further we recall some notation and terminology. By $l^{\infty}$ and $c$ we denote the spaces of all bounded and convergent real sequences, respectively.

Let $T=\left(t_{n k}\right)$ be an infinite matrix, and let $X$ and $Y$ be two sequence spaces. If $T x$ exists for each $x \in X$ and $T x \in Y$ then we say that $T$ maps $X$ into $Y$. The set of matrices which map $X$ into $Y$ is denoted by $(X, Y)$. The set of matrices which map $X$ into $Y$ and leave the limit or sum invariant is denoted by $(X, Y ; p)$. For example, if $T \in(c, c ; p)$, then $\lim T x=\lim x$ for every $x \in c$. In this case $T$ is called regular (see [1], [11]). If it is regular and satisfies

$$
\lim _{n} \sum_{k}\left|t_{n k}-t_{n, k+1}\right|=0,
$$

then $T$ is called strongly regular [11].

Received by the editors Oct. 25, 2008, Accepted: Dec. 25, 2008.

2000 Mathematics Subject Classification. Primary 40A05.

Key words and phrases. almost convergent sequence, core of a sequence. 
It is well-known [7], [11] that the functional

$$
q(x)=\inf _{n_{1}, n_{2}, \ldots n_{r}} \limsup _{k} \frac{1}{r} \sum_{i=1}^{r} x_{k+n_{i}}
$$

is sublinear on $l^{\infty}$. We consider the following functionals on $l^{\infty}$ :

$$
\begin{aligned}
& L(x)=\limsup x_{n}, \\
& l^{*}(x)=\liminf _{r} \sup _{k} \frac{1}{r} \sum_{i=0}^{r} x_{k+i}, \\
& L^{*}(x)=\limsup _{r} \sup _{k} \frac{1}{r} \sum_{i=0}^{r} x_{k+i} .
\end{aligned}
$$

It follows from the Corollary of Theorem 1 in [3] that $q(x)=L^{*}(x)$.

If $q(x)=-q(-x)=s$, then $x$ is called almost convergent to $s$ [7], and in this case we write $F-\lim x=s$. By $F$ we denote the set of all almost convergent sequences.

The Banach core $(\mathcal{B}$-core) of a bounded sequence $x$ is defined to be the closed interval $[-q(-x), q(x)]$ (see [2], [6]). Since $q(x) \leq L(x)$ for every $x \in l^{\infty}$, it follows that $\mathcal{B}$-core $\{x\} \subseteq \mathcal{K}$-core $\{x\}$ where $\mathcal{K}$-core $\{x\}$ is the Knopp core and it is given by $\mathcal{K}$-core $\{x\}=[\lim \inf x, \lim \sup x]$. It is shown in [6], [10] that

$$
\mathcal{K}-\operatorname{core}\{A x\} \subseteq \mathcal{B}-\operatorname{core}\{x\} \text { ( for every } x \in l^{\infty} \text { ) }
$$

if and only if $A$ is strongly regular and $\lim _{n} \sum_{k}\left|a_{n k}\right|=1$.

With this terminology the Knopp core theorem gives the conditions on the matrix $A$ so that the inequality $L A \leq L^{*}$, on $l^{\infty}$, holds. Hence (1) yields the inequality $L A \leq L^{*}$ on $l^{\infty}$.

Also it is well-known [8], [3] that the functional

$$
Q(x)=\inf _{n_{1}, n_{2}, \ldots n_{r}} \limsup _{k} \frac{1}{r} \sum_{i=1}^{r}\left|x_{k+n_{i}}\right|
$$

is sublinear on $l^{\infty}$. Define for $x \in l^{\infty}$,

$$
L^{* *}(x)=\limsup _{r} \sup _{k} \frac{1}{r} \sum_{i=0}^{r}\left|x_{k+i}\right| .
$$

Then substituting $|x|=\left(\left|x_{n}\right|\right)_{n \geq 0}$ for $x=\left(x_{n}\right)$, in Corollary of Theorem 1 in [3], we obtain $Q(x)=L^{* *}(x)$

Throughout the paper we consider only real matrices and real bounded sequences.

In this paper we will give a reception between functionals $L$ and $L^{* *}$, than some inequalities. 


\section{THE FUNCTIONALS $L^{* *}$ AND $l^{* *}$ AND SOME INEQUALITIES}

If we take the sequence $x=\left(x_{n}\right)$ defined by $x_{n}=(-1)$ for all $n$, it follows that,

$$
L(x)=-1, L^{* *}(x)=1
$$

hence

$$
L^{* *}(x)>L(x) .
$$

Now, if we define sequence $x=\left(x_{n}\right)$ by $x_{n}=(-1)^{n}$ for all $n$, it follows that,

$$
L(x)=1, L^{* *}(x)=1,
$$

hence

$$
L^{* *}(x)=L(x) .
$$

Finally, if we consider the bounded sequence $x=\left(x_{n}\right)$ given by $x_{n} \geq 0$ for all $n$, then

$$
L^{* *}(x)=L^{*}(|x|)=L^{*}(x) \leq L(x),
$$

hence

$$
L^{* *}(x) \leq L(x) .
$$

In this paper we mainly compare $L A$ with $L^{* *}$.

Theorem 2.1. If $L^{* *}(x-y)=0$ on $l^{\infty}$, then $L^{* *}(x)=L^{* *}(y)$

Proof. We know that

$$
L^{* *}(x-y)=\limsup _{r} \sup _{k} \frac{1}{r} \sum_{i=0}^{r}\left|x_{k+i}-y_{k+i}\right| .
$$

Now

$$
\begin{aligned}
& L^{* *}(x)=\limsup _{r} \sup _{k} \frac{1}{r} \sum_{i=0}^{r}\left|x_{k+i}\right| . \\
& =\limsup _{r} \sup _{k} \frac{1}{r} \sum_{i=0}^{r}\left|x_{k+i}-y_{k+i}+y_{k+i}\right| \\
& \leq \quad \limsup _{r} \sup _{k} \frac{1}{r} \sum_{i=0}^{r}\left|x_{k+i}-y_{k+i}\right|+\limsup _{r} \sup _{k} \frac{1}{r} \sum_{i=0}^{r}\left|y_{k+i}\right| \\
& =0+\limsup _{r} \sup _{k} \frac{1}{r} \sum_{i=0}^{r}\left|y_{k+i}\right| \\
& =L^{* *}(y)
\end{aligned}
$$

If we interchange the roles of $x$ and $y$,then we also get

$$
L^{* *}(y) \leq L^{* *}(x)
$$

which implies that

$$
L^{* *}(x)=L^{* *}(y)
$$


Theorem $1 \mathrm{in}[5]$ is valid if we write $\left(\left|x_{n}\right|\right)$ in place of $x \in l^{\infty}$. Using this result we get,

$$
\limsup _{n} \sup _{i} \frac{1}{n+1} \sum_{r=i}^{i+n}\left|x_{r}\right| \leq L(|x|) .
$$

If we define sublinear functional $P$ on $l^{\infty}$ by

$$
P(x)=\limsup _{k}\left|x_{k}\right|,
$$

we can give the following

Corollary 1. On $l^{\infty}$,

$$
L^{* *} \leq P
$$

Let us define the functional $Z$ on $l^{\infty}$ by

$$
Z(x)=\frac{\left|x_{1}\right|+\left|x_{2}\right|+\ldots+\left|x_{k}\right|}{k} .
$$

We recall that the matrix $B$ called normal if it is lower semi triangular matrix with non-zero diagonal entries.

Theorem 7 of Yardimci [13] gives us the necessary and sufficient conditions for $L^{*}(A x) \leq L(B x)$, whenever $B$ is a normal matrix and $B x$ is bounded. This theorem is valid if we take $C_{1}$ Cesáro matrix instead of $B$ and $|x|=\left(\left|x_{n}\right|\right)$. Thus we get the following

Corollary 2. On $l^{\infty}$,

$$
L^{* *} A(x) \leq Z(x) .
$$

The following result compares $L A$ with $L^{* *}$.

Theorem 2.2. If $A$ is a strogly regular matrix and

$$
\lim _{n} \sum_{k}\left|a_{n k}\right|=1
$$

then

$$
L A \leq L^{* *}
$$

on $l^{\infty}$.

Proof. Let $A$ be a strongly regular matrix and $\lim _{n} \sum_{k}\left|a_{n k}\right|=1$. Then Theorem 6 [10] implies that

$$
L A(x) \leq L^{*}(x)
$$

for all $x \in l^{\infty}$. Also we know that,

$$
L^{*}(x) \leq L^{*}(|x|)=L^{* *}(x)
$$

on $l^{\infty}$. So,

$$
L A(x) \leq L^{* *}(x)
$$

on $l^{\infty}$. This proves the theorem. 
The following theorem also gives some sufficient conditions for this inequality.

Theorem 2.3. Let $A$ be a strongly regular matrix. Then If there exist a nonnegative strongly regular matrix $B$, which is absolutely equivalent to $A$ on $l^{\infty}$, then

$$
L A(x) \leq L^{* *}(x),\left(\text { for every } x \in l^{\infty}\right) .
$$

Proof. By absolute equivalence of $A$ and $B$, for every $x \in l^{\infty}$,

$$
\lim _{n}\left\{(A x)_{n}-(B x)_{n}\right\}=0 \text {. }
$$

Now Theorem 6.5.I of Cooke [1] implies that

$$
L(A x) \leq L(x),\left(\text { for every } x \in l^{\infty}\right) .
$$

Since $B$ is non-negative strongly regular matrix, it follows from Theorem 3 in [10] that, for every $x \in l^{\infty}$,

$$
L(B x) \leq L^{*}(x) .
$$

Since (2) holds, Theorem 6.3.II of Cooke [1] implies that

$$
L(A x)=L(B x) \text {. }
$$

Now (3) and (4) imply

$$
L(A x) \leq L^{*}(x) \leq L^{* *}(x) .
$$

Define the functionals $l^{* *}$ on $l^{\infty}$ by

$$
l^{* *}(x)=\liminf _{r} \sup _{k} \frac{1}{r} \sum_{i=0}^{r}\left|x_{k+i}\right| .
$$

With this definition we have

Theorem 2.4. Let $A$ be any matrix such that $\sup _{n} \sum_{k}\left|a_{n k}\right|<\infty$. If

$$
\limsup _{n} \sup _{i} \frac{1}{n+1} \sum_{r=i}^{i+n}\left|a_{r k}\right|=0,
$$

then we have $L^{* *} A \leq l^{* *}$ on $l^{\infty}$.

Proof. By hypothesis, $A x$ exist for every $x \in l^{\infty}$. Then,

$$
\begin{aligned}
& L^{* *}(A x)=\limsup _{n} \sup _{i} \frac{1}{n+1} \sum_{r=i}^{i+n}\left|\sum_{k} a_{r k} x_{k}\right| \\
& \leq\|x\| \limsup _{n} \sup _{i} \frac{1}{n+1} \sum_{r=i}^{i+n} \sum_{k}\left|a_{r k}\right| \\
& =\|x\| \lim \sup _{n} \sup _{i} \sum_{k} \frac{1}{n+1} \sum_{r=i}^{i+n}\left|a_{r k}\right|=0 .
\end{aligned}
$$

Also we know that

$$
L^{* *}(A x) \geq 0
$$


Hence we get

$$
L^{* *}(A x)=0
$$

From the definition of $l^{* *}$ we can write

$$
l^{* *}(x) \geq 0
$$

So we get

$$
L^{* *}(A x) \leq l^{* *}(x)
$$

ÖZET: Bu çalışmada temel amacımız, sınırlı reel diziler üzeinde tanımlı $L^{* *}$ ve $l^{* *}$ fonksiyonellerini incelemek ve bunlar arasındaki bazı eşitsizlikleri vermektir.

\section{REFERENCES}

[1] R.G. Cooke, Infinite Matrices and Sequence Spaces, Macmillan, 1950.

[2] G. Das, Sublinear functionals and a class of conservative matrices, Bull. Inst. Math. Acad. Sinica 15 (1987), 89-106.

[3] G. Das and S.K. Mishra, A note on a theorem of Maddox on strong almost convergence, Math. Proc. Camb. Phil. Soc. 89 (1981), 393-396.

[4] M. Jerison, The set of all generalized limits of bounded sequences, Canad. J.Math. 9 (1957), 79-89.

[5] K. Knopp, Zur theorie der limitierungsverfahren(Erste Mitteilung), Math. Z. 31 (1930), 97127.

[6] L. Loone, Knopp's core and almost-convergence core in the space m, Tartu Riikl. All. Toimetised 335 (1975), 148-156.

[7] G.G. Lorentz, A contribution to the theory of divergent sequences, Acta Math. 80 (1948), $167-190$.

[8] I.J. Maddox, A new type of convergence, Math. Proc., Cambridge Philos. Soc. 83(1978), 61-64.

[9] I.J. Maddox, Some analogues of Knopp's core theorem, Internat. J. Math.\& Sci. 2 (1979), 605-614.

[10] C. Orhan, Sublinear functionals and Knopp's core theorem, Internat. J. Math.\&Math. Sci. 13 (1990), 461-468.

[11] G.M. Petersen, Regular Matrix Transformations. London, McGraw-Hill, 1966.

[12] S. Simons, Banach limits, infinite matrices and sublinear functionals, J. Math. Anal. Appl. 26 (1969) 640-655.

[13] S. Yardimci, Core theorems for real bounded sequences, Indian J. Pure Appl. Math. 27 (1996), 861-867.

Current address: Department of Mathematics

E-mail address: yardimci@science.ankara.edu.tr

$U R L:$ http://math.science.ankara.edu.tr 


\section{INSTRUCTIONS TO CONTRIBUTORS}

Mathematics and Statistics of COMMUNICATIONS accepts original research articles written in English in the fields of Mathematics and Statistics. Review articles written by eminent scientists can also be invited by the Editor.

Three copies of the manuscripts must be submitted in AMS Article Tex format.

Formulas should be numbered consecutively in parentheses ( ) and each rnanuscript should be accompanied by classification numbers from the American Mathematical Society's classification scheme.

It is a fundamental condition that articles submitted to Communications have not been previously published and will not be simultaneously submitted or published elsewhere. After the manuscript has been accepted for publication, i.e., after referree-recommended revisions are complete, the author will not be permitted to rnake any new additions to the manuscript.

Before publication the galley proof is always sent to the author for correction. Thus it is solely the author's responsibility for any typographical mistakes which occur in their article as it appears in the Journal.

\section{Title Page:}

The title should not be long but should be informative. Each title page must contain (i) The title of the paper in English, (ii) The complete name(s) of the author(s), (iii) The name and the address of the University.

\section{Abstract :}

The abstract should not exceed 200 words and it should condense the essential features of the articles with the focus on the major advances in the field.

\section{References :}

References must be listed in alphabetical order at the end of the article and then numbered in brackets [1]. Within the manuscript, refer to the references by their given number, again in brackets [1]. They should be styled and punctuated according to the following examples:

[1] Kelley, J.L., General Topology, 1970, New York: Van Nostrand.

[2] Maddox,1.J., Some incusion theorems, Proc. Glasgow Math. Assn. 6 (1964),

161-168.

Abstracts, unpublished data and personal communications should not be given in the references but they may be mentioned in the text.

\section{Footnotes:}

Footnotes, except a possible acknowledgement of assistance or financial support on the first page, should be avoided by being incorparated into the text. 


\section{Tables and Figures:}

All tables and figures must be numbered consecutively throughout the paper ( Table 1, Figure 2) and also have a caption or legend.

\section{Acknowledgements:}

Acknowledgements should be given as short as possible at the end of the text.

7. Reprints:

25 free reprints will be provided for each paper. Irrespective of their acceptance, manuscripts will not be returned to the authors.

8. Computer Disk:

After the acceptance of manuscripts for publication, we will ask you to submit a revised electronic copy of the manuscript which is written in AMS tex format. For electronic submission please visit our WEB page at http://math.science.ankara.edu.tr/dergi/dergi.htm

\section{Charges:}

Each paper is due to be charged for the amount of which is determined by the administiration each year.

\section{Address:}

Text should be sent to the following address:

Prof.Dr. Öner ÇAKAR- Editor-in-Chief, Communications

Ankara University, Faculty of Sciences

06100, Tandoğan, ANKARA - TURKEY 